\title{
Effect of Bacillus pumilus 3-19 protease on growth parameters and gut microbiome of broiler chickens.
}

\author{
Daria Pudova ${ }^{1, *}$, Anastasia Koryagina ${ }^{1}$, Natalya Rudakova ${ }^{1}$, Ayslu Mardanova ${ }^{1}$ and \\ Margarita Sharipova ${ }^{1}$ \\ ${ }^{1}$ FSAEI HE Kazan (Volga region) Federal University, Institute of Fundamental Medicine and \\ Biology, 420008, 18 Kremlevskaya Str., Kazan, Russia
}

\begin{abstract}
The objective of the study was to determine the effect of adding protease obtained from the Bacillus pumilus 3-19 strain to the diet of Hubboard broiler chickens on the growth indicators of poultry and the composition of the cecum microbiota of broiler chickens. As a result of metagenomic analysis of 16S rRNA genes in the contents of the cecum of chickens, it was shown that the control and experimental groups formed two separate clusters, which indicates differences in microbial communities in these two groups. Analysis of alpha diversity showed an increase in species richness in the group of chickens that received protease as a feed additive. On day 35 , both groups were dominated by bacterium of Firmicutes (21.13 - 49.01\%) and Bacteroidetes (37.68 - 67.72\%) groups. It was shown that in the group of chickens receiving protease as a feed additive, the average daily gain in live weight was higher than in the control group by $0.5 \mathrm{~kg}(\mathrm{P}<0.05)$. The introduction of protease resulted in an increased $\mathrm{F} / \mathrm{B}$ ratio, which was positively correlated with chicken body weight. Thus, the addition of B. pumilus 3-19 protease to broiler feed leads to improved feed intake and digestibility, which is of great importance for reducing the cost of the final product. The use of protease regulates the cecum microbiota of broiler chickens and increases microbial diversity on 35 day of chicken growth.
\end{abstract}

\section{Introduction}

The expected expansion of animal husbandry, due to the growth of the world's population, creates a global shortage of feed protein. Due to this, feed protein has become one of the most expensive and limiting feed additives [1]. It is known that biological fermentation of protein feeds, reducing the amount of dietary protein using amino acids, and the use of proteases will help to solve this problem or, at least, reduce the supply and demand deficit [2]. Proteases are among the three largest groups of industrial enzymes and account for about $60 \%$ of the total sales of enzymes in the world, $40 \%$ of which are bacterial proteases [3]. Proteases have the potential to improve the growth of poultry, since the activity of pancreatic proteases in chickens is reduced [4]. Exogenous proteases can

* Corresponding author: dasha171711@gmail.com 
complement pancreatic enzymes and increase the rate of intestinal protein degradation [5]. In addition to improving the digestibility of proteins, adding proteases to feed increases the absorption of amino acids by animals and saves money on the purchase of synthetic amino acids [6]. It has been demonstrated that exogenous enzymes can affect the microbiome of the gastrointestinal tract of chickens, the composition of which has a significant impact on the poultry health and growth indicators [7]. Exogenous proteases can alter the degree of degradation of feed substrates in the broiler digestive system and potentially alter the nutrients used by the digestive tract microbiota [8]. Most studies of proteases use were conducted in combination with other enzymes, while several studies were performed with monocomponent proteases, and these studies gave different results $[9,10]$. In addition, only a few studies have studied the effect of exogenous enzymes on the chicken microbiome using metagenomic analysis of the 16S rRNA gene [11,12]. Thus, the objective of this study was to study the effect of exogenous protease on poultry growth rates and changes in the digestive tract microbiota. Our hypothesis was that the protease would change the gut microbiota of broilers and ultimately improve growth rates during the first 35 days of their life.

\section{Methods of research}

\subsection{Creation of the enzyme}

Subtilisin-like proteinase was isolated and purified using the B. pumilus 3-19 strain, as described earlier [13].

\subsection{Animals and diet}

All animals were kept in accordance with the Guidelines for the care and use of experimental animals of the Kazan Federal University and a commercial poultry farm (PFE Alimchueva Z.I., Russia, the Republic of Mari El, Medvedevsky district, village of Srednyaya Azyakovo). 180-day-old broiler chickens (Hubboard) were purchased in an industrial incubator with an average weight of $22 \pm 0.21 \mathrm{~g}$. Chickens were weighed individually, labeled using plastic rings of different colors, and randomly assigned to 2 groups (control and experimental) with 3 repetitions and 30 chickens per repetition. The birds were kept in three-tiered cages made of galvanized mesh $(0.5 \times 0.5 \mathrm{~m} \times 0.35 \mathrm{~m}$ for 5 birds) with controlled temperature conditions. The initial temperature was $32^{\circ} \mathrm{C}$ in the first week of age and gradually decreased by $2^{\circ} \mathrm{C}$ per week until it reached about $18^{\circ} \mathrm{C}$ at the end of the experiment. The lightning period of 23 hours per day was provided throughout the entire experimental period. Chickens were allowed free access to water and feed during the experimental period. The basic diet (BD) did not contain any enzyme supplements (control group). For the experimental group, the BD was supplemented with purified subtilisin-like protease $(10 \mathrm{u} / \mathrm{kg})$ from the sixth day (experimental group). Broilers were fed in abundance using dry mixed feeds with nutrition parameters that meet the recommended feeding standards (GOST 18221-99 "Mixed feed for poultry. Technical conditions").

\subsection{Growth indicators}

Chickens were individually weighed to the nearest gram at the beginning of the experiment and on $1,8,15,22,28$, and 35 days. The safety of dietary supplements for poultry was determined by monitoring daily mortality in each group of poultry. To study the effect of proteinase supplementation on the digestibility and assimilation of nutrients, a balance 
experiment was performed. The poultry was kept in separate cages with a mesh bottom, under which frames made of plastic film were installed to collect droppings. The balance experience is divided into two periods: preliminary - 5 days, accounting -3 days. The experiment takes into account individually for each bird: the amount and chemical composition of the feed consumed and the excreted droppings. The droppings are collected twice a day (morning and evening), weighed, placed in double plastic bags (carefully closed), filled with $0.1 \mathrm{n}$ oxalic acid solution ( $2 \mathrm{ml}$ per $50 \mathrm{~g}$ of droppings) to bind ammonia. The amount of acid released is taken into account when determining the initial water. The chemical composition of the feed mixture, droppings will be carried out in the laboratory of chemical analysis of feed using the methods described in GOST 31640-2012, 32933-2014 and 31675-2012 (GOST 31640-2012 "Feed. Methods of dry matter content determination" GOST 32933-2014 "Feed, compound feed. Method of crude ash determination", GOST 31675-2012 "Feed. Methods for determining the content of crude fiber with intermediate filtration", the Kjeldahl method for determining nitrogen, the Soxlet extraction method for fat determination). The digestibility coefficient was calculated as the ratio of digested to digestible nutrients, expressed as a percentage.

Body weight (BW) and average daily weight gain (ADWG) of broilers were measured on day 35 of chickens' growth. Feed intake (FI) was evaluated weekly. FI was then reevaluated for one bird. The feed conversion rate (FCR) was obtained on the 42nd day of age, and the European broiler productivity index (EPI) was calculated using the formula:

$$
E P I=\frac{\text { Viability }(\%) \times B W(\mathrm{~kg}) \times 100}{\text { Age }(\mathrm{d}) \times F C R(\text { kgfeedintake } / \text { kggain })}
$$

Room temperature and relative humidity were also recorded daily and adjusted accordingly to avoid the impact of stressful conditions on broiler chickens.

\subsection{Sample preparation, DNA isolation and 16S rRNA gene sequencing}

To determine the effect of feed protease on the broiler chickens microbiome, 7 birds from the control group and 8 birds from the experimental group were selected. All birds were killed on 35 day of the experiment. Immediately after euthanasia, each bird's cecum was cut open, the contents were collected in a $3 \mathrm{ml}$ sterile tube, frozen with liquid nitrogen, and delivered to the laboratory in a dry ice bag, then stored at $-80^{\circ} \mathrm{C}$ until the DNA was isolated. Genomic DNA was isolated from the contents of the cecum using a commercial QIAamp Fast DNA Stool Mini kit (QIAGEN, Germany). The concentration and quality of DNA were evaluated using NanoDrop 2000 (Thermo, USA) and gel electrophoresis. PCR libraries were created using universal primers 341F (5-CCT ACG GGN GGC WGC AG-3) and 805R (5-GAC TAC HVG GGT ATC TAA TCC-3) targeting variable regions V3 - V4 of the bacterial 16s rRNA gene. Sequencing was performed on the Illumina MiSeq platform using the MiSeq v3 reagent kit (Illumina, USA) at the KFU-Riken Laboratory of the Kazan Federal University (city of Kazan, Russia).

\subsection{Sequencing data processing and statistical analysis}

Libraries obtained as a result of sequencing by the method of paired reads were analyzed using the QIIME software package version 1.9.1. Reads with a quality lower than Q20 were removed from further analysis. The presence of chimeric sequences was performed using the usearch61 method from the Greengenes 13_8 database. After qualitative filtering and elimination of chimeric sequences, the resulting "reads" were grouped into operational taxonomic units (OTU) with $97 \%$ sequence similarity. At the same 
time, the minimum size of the OTU required to save it was equal to 5 sequences. The RDP version 2.2 database was used for taxonomic classification of reads. Alpha diversity indexes were calculated using the QIIME "alpha_rarefaction.py" script. The following indexes were calculated: the Chaol diversity index and the number of OTU observed. The beta diversity between all pairs of samples was calculated using the QIIME "beta_diversity_through_plots.py" script. The results for the weighted UniFrac distances were visualized with the EMPeror. Comparisons between groups were performed using variance analysis in GraphPad program (USA). The results were presented as an average value \pm standard deviation, considering the $p \leq 0.05$ value to be significant.

\section{Research results and discussion}

\subsection{Overall performance}

Body weight and average daily growth are one of the most important zootechnical indicators of production activity in any livestock and poultry industry. Periodic monitoring of changes in body weight allows specialists to identify technological shortcomings in a separate age period of young animals growing and to eliminate them. In our studies, the control of changes in the body weight of broilers was carried out during the entire accounting period with a seven days frequency. On day 35 , the average daily gain was higher in broilers receiving a basal diet with added proteinase than in broilers receiving only a basal diet $(\mathrm{P}<0.05$, Table 1$)$. Compared with the control, the average daily feed intake significantly decreased in broilers that received basal diets with the addition of proteinase $(\mathrm{P}<0.05)$.

By the end of the experiment, after 5 weeks, the maximum body mass index was recorded in the experimental group $(1550.7 \pm 6.52 \mathrm{~g})$, which is $4.2 \%$ more than in the control group (Table 1). A similar pattern was observed in the analysis of absolute growth. The data obtained correspond to studies of Murugesan et al. [14], which showed that exogenous proteases can enhance the effect of endogenous peptidases by increasing protein digestibility and hydrolysis of protein anti-nutritional factors such as lectins, trypsin inhibitors, and antigenic proteins [14].

Table 1. Growth indicators for broiler chickens

\begin{tabular}{|l|c|c|c|}
\hline \multirow{2}{*}{ Parameters } & \multicolumn{2}{|c|}{ Diet } & \multirow{2}{*}{-value } \\
\cline { 2 - 3 } & Control group & Experimental group & \\
\hline Absolute growth (g) & $1465.9 \pm 37.4$ & $1528,7 \pm 42.0$ & 0.004 \\
\hline Feed consumption (g) & $2521 \pm 7.48$ & $2440 \pm 8.01$ & 0.0083 \\
\hline Feed conversion rate & $1.75 \pm 0.078$ & $1.61 \pm 0.041$ & 0.05 \\
\hline European productivity index & $242,94 \pm 5.10$ & $275,06 \pm 2.84$ & 0.0016 \\
\hline
\end{tabular}

Table 2. The effect of adding protease on digestibility

\begin{tabular}{|l|c|c|c|}
\hline $\begin{array}{l}\text { Digestibility coefficient, } \\
\text { \% }\end{array}$ & Control group & Experimental group & $\boldsymbol{p}$-value \\
\hline Protein & $74 \pm 0.6$ & $81.1 \pm 0.5$ & 0.0005 \\
\hline Dry extract & $72.6 \pm 0.9$ & $75.5 \pm 1.1$ & 0.0003 \\
\hline Fibers & $10.3 \pm 0.4$ & $12.4 \pm 0.2$ & 0.0001 \\
\hline Fats & $57.4 \pm 0.9$ & $60.1 \pm 1.1$ & 0,0001 \\
\hline
\end{tabular}

The digestibility of nutrients and feed entering the body largely depends on the enzymatic activity of the internal secretion glands, secretory function of the gastrointestinal 
tract and individual organs. The increase in the digestibility of organic matter in the diet of broiler chickens of the experimental group was mainly due to the digestibility of fat and protein. The highest protein digestibility was observed in the experimental group and was $81.1 \pm 0.5 \%$, which is $7.1 \%$ higher than in the control group (Table 2). The protein digestibility coefficient in the experimental group was higher $(\mathrm{P}<0.05)$ compared to the control by $7.1 \%$. The digestibility of dry extract in the control was $72.6 \pm 0.9 \%$, and in the experimental group $-75.5 \pm 1.1 \%$. In the experimental group, a higher fiber digestibility coefficient was observed $(12.4 \pm 0.2)$. The fat absorption coefficient was $57.4 \pm 0.9 \%$ (control group) and $60.1 \pm 1.1 \%$ (experimental group) (Table 2). The results are consistent with a study of Freitas et al. [15], the effects of live performance associated with the addition of protease occurred in parallel with an increase in protein and fat digestibility.

\subsection{Analysis of chicken cecum microbiota}

On average, 126,117 reads were obtained for each sample of the cecum of birds, of which 72,464 sequences remained as a result of filtering. The resulting reads were grouped in OTU with a 97\% similarity. At the same time, $1012 \pm 106$ and $1193 \pm 117$ OTU were identified for the control and experimental groups, respectively. The alpha diversity of the chicken gut microbial community was evaluated by the number of OTU observed and the Chaol diversity index. The addition of protease to the feed resulted in an increase in the number of observed OTU from $890.56 \pm 56.06$ to $1052.25 \pm 97.59$ ( $p \leq 0.05)$. Another index of microbial diversity (ChaoI index) was also higher in chickens receiving a diet with added protease $(1294.57 \pm 107.24$ for the control and $1579.02 \pm 105.19$ for the experiment $(p \leq 0.05))$, indicating an increase in species diversity. The analysis of the main coordinates describing the $\beta$-diversity showed a marked differentiation between the microbial populations of chickens in the control group and the group that received protease as a feed additive (Figure 1).

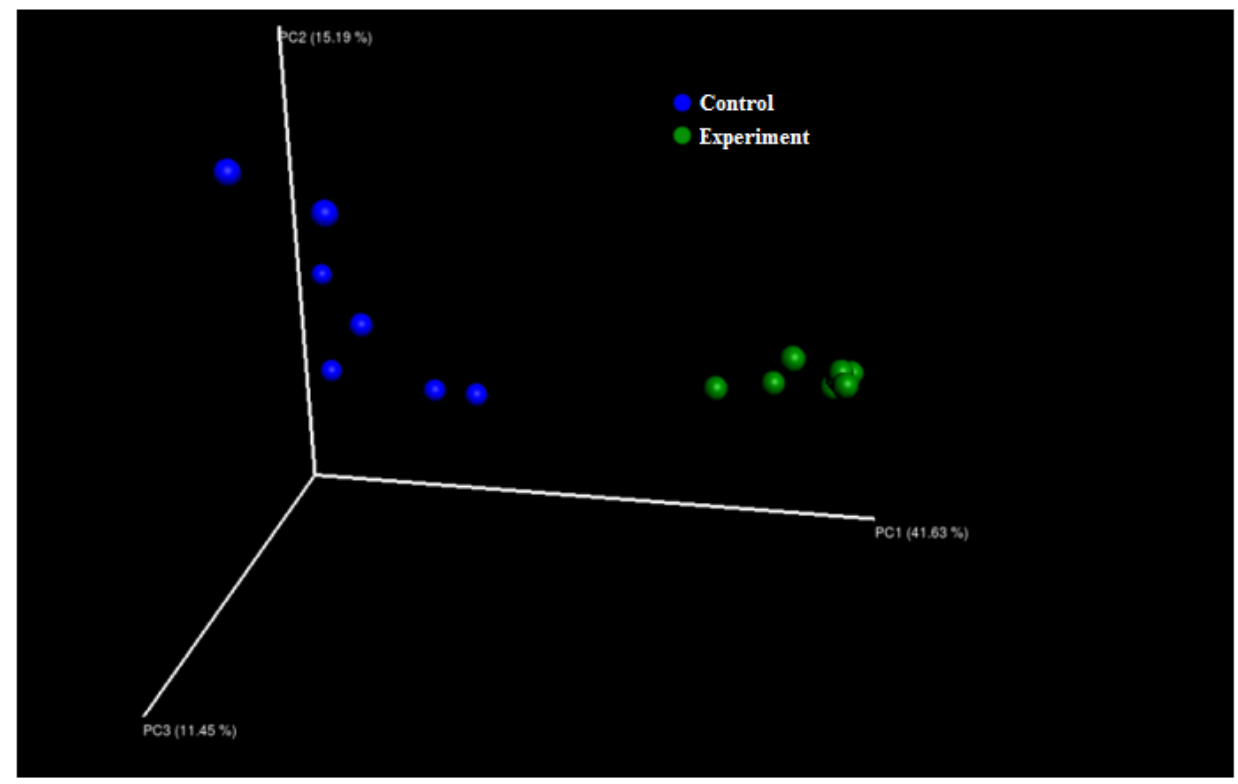

Fig. 1. Graph of principal component analysis (PCoA) based on weighted Unifrac distances between the cecum microbial communities of broilers treated with protease (experience) and broilers treated with a diet without protease (Control). The PCoA graph confirmed differences in the microflora of the experimental and control groups, depending on the diet. 


\subsection{Analysis of the microbial composition of chicken gut microbiota}

The analysis of cecum samples from both groups identified 11 types, 21 classes, 26 orders, 39 families, and 69 genuses. As shown in Table 3, Bacteroidetes (37.68-67.72\%) had the highest relative abundance, followed by Firmicutes (21.13-49.01\%), Proteobacteria (2.01$3.99 \%)$ and Actinobacteria $(0.24-0.43 \%)$. The presence of protease in the diet led to a decrease in the presence of Bacteroidetes and an increase in the number of representatives of the Firmicutes type. Two dominant types of bacteria, Bacteroidetes and Firmicutes, are known to play an important role in the digestion of nutrients. An increase in Firmicutes may lead to greater nutrient uptake, whereas an increase in Bacteroidetes may reduce nutrient uptake [11]. However, many genes involved in the metabolism and degradation of complex polysaccharides and monosaccharides have been identified in the Bacteroidetes genome. These genes actively produce organic acids, as well as encode proteins and enzymes that play a role in the digestion of nutrients [16]. In the group of chickens receiving protease as a feed additive, the ratio of Firmicutes/Bacteroidetes $(\mathrm{F} / \mathrm{B})$ significantly increased (from 0.3 to 1.3 ). It has been shown that an increased F/B ratio in the cecum leads to active fermentation of volatile fatty acids, which promotes fat deposition and stimulates chicken growth [17].

Table 3. Effect of protease on the relative abundance of the main types of bacteria

\begin{tabular}{|l|l|l|}
\hline Type & Control, \% & $\begin{array}{l}\text { Experiment, } \\
\%\end{array}$ \\
\hline Bacteroidetes & $67.72 \pm 6.26$ & $37.68 \pm 5.74$ \\
\hline Firmicutes & $21.13 \pm 3.40$ & $49.01 \pm 6.89$ \\
\hline Proteobacteria & $2.01 \pm 1.70$ & $3.99 \pm 2.15$ \\
\hline Actinobacteria & $0.43 \pm 0.21$ & $0.24 \pm 0.14$ \\
\hline Genus & & \\
\hline Streptococcus & $0.05 \pm 0.01$ & $0.12 \pm 0.02$ \\
\hline Enterococcus & $0.01 \pm 0.02$ & $0.13 \pm 0.01$ \\
\hline Lactobacillus & $0.35 \pm 0.03$ & $0.21 \pm 0.02$ \\
\hline Clostridium IV & $0.14 \pm 0.07$ & $0.13 \pm 0.01$ \\
\hline Clostridium XI & $0.01 \pm 0.01$ & $0.02 \pm 0.00$ \\
\hline Clostridium XIVa & $0.58 \pm 0.09$ & $0.21 \pm 0.08$ \\
\hline Bacteroides & $44.63 \pm 0.08$ & $30.04 \pm 0.12$ \\
\hline
\end{tabular}

The addition of protease to feed resulted in an increase in the proportion of Streptococcus and Enterococcus in the intestines of broiler chickens (Table 3). Representatives of the Streptococcus genus are associated with an increase in the density of CD8+ T-cells that affect immune functions in the gut and may be involved in reducing the number of pathogens [18]. Enterococcus is usually found in small numbers in the small intestines of broiler chickens [19]. It was proved that the probiotic mixture of Enterococcus and Lactobacillus increased the number of bacteria attached to the mucosa in the terminal part of the small intestine [20]. In addition, strains of this genus are able to synthesize bacteriocins that are active against pathogens such as Eimeria spp., which makes these bacteria potential candidates for probiotics [21]. The proportion of representatives of the Clostridium genus did not show significant changes depending on the use of protease in broiler feeding (Table 3). Species belonging to Clostridium IV and XI clusters are able to 
increase the growth of chickens by producing butyrate, which is an indispensable source of energy for the intestinal wall and a mediator of immune responses [22].

In this study, the addition of protease promoted poultry growth and regulated the gut microbiota, in particular, increasing the $\mathrm{F} / \mathrm{B}$ ratio in the experimental group compared to the control.

\section{Conclusion}

The addition of protease to feed improved poultry growth rates and caused some changes in the diversity of the broiler gut microbiota. The presence of protease in the feed improved protein absorption, which increased the live body weight of broiler chickens by $0.5 \mathrm{~kg}$, and also reduced feed consumption in the experimental group. Metagenomic analysis of $16 \mathrm{~S}$ rRNA genes of the contents of the cecum of broilers showed an increase in microbial diversity and the ratio of Firmicutes/Bacteroidetes in the experimental group compared to the control. Thus, the addition of B. pumilus subtilisin-like proteinase has a beneficial effect on the growth and availability of nutrients by broilers, as well as a positive effect on the intestinal microflora of birds.

\section{Acknowledgment}

This work was supported by grant of the RSF № project 16-16-04062.

\section{References}

1. S.W. Kim, J.F. Less, L. Wang, T. Yan, V. Kiron, S.J. Kaushik, X.J. Lei, Annual Review of Animal Biosciences, 7, 221-243 (2019)

2. F.L. Law, I. Zulkifli, A.F. Soleimani, J.B. Liang, E.A. Awad, Asian-Australas J Anim Sci, 31, 1291-1300 (2017)

3. K. Rani, R. Rana, S. Datt, Int J Curr Life Sci, 2, 12-18 (2012)

4. S.H. Jin, A. Corless, S.L. Sell, Worlds Poult. Sci. J., 54, 335-345 (1998)

5. T. Mahmood, M.A. Mirza, H. Nawaz, M. Shahid, Livest Sci, 200, 71-75 (2017)

6. M. Toghyani, C.K. Girish, S.B. Wu, P.A. Iji, R.A. Swick, Poultry Science, 96, 12681279 (2017)

7. A.J. Cowieson, J. Poult. Sci., 47, 1-7 (2010)

8. E. Kiarie, L.F. Romero, C.M. Nyachoti, Nutr. Res. Rev., 26, 71-88 (2013)

9. C.R. Angel, W. Saylor, S.L. Viera, N. Ward, Poult. Sci., 90, 2281-2286 (2011)

10. C.L. Walk, K. Juntunen, M. Paloheimo, D.R. Ledoux, Poult. Sci., 98, 5525-5532 (2019)

11. Y. Xiao, Y. Xiang, W. Zhou, J. Chen, K. Li, H. Yang, Poult. Sci. 96, 1387-1393 (2017)

12. P.M. Munyaka, N.K. Nandha, E. Kiarie, C.M. Nyachoti, E. Khafipour, Poult. Sci., 95, 528-540 (2016)

13. A.O. Koryagina, N.L. Rudakova, M.T. Lutfullin, G.F. Khadieva, A.A. Toymentseva, A.M. Mardanova, M.R. Sharipova, Agricultural Biology, 53, 6 (2018)

14. G.R. Murugesan, B. Syed, S. Haldar, M.E. Persia, Front Vet Sci, 2, 21 (2015)

15. D.M. Freitas, S.L. Vieira, C.R. Angel, A. Favero, A. Maiorka, J Appl Poult Res, 20, 3 (2011) 
16. M. Medvecky, D. Cejkova, O. Polansky, D. Karasova, T. Kubasova, A. Cizek, BMC Genomics, 19, 561 (2018)

17. S. Salaheen, S.W. Kim, B.J. Haley, J.A.S. Van Kessel, D. Biswas, Front. in Microbiol, 8, 2088 (2017)

18. P. Huang, Y. Zhang, K. Xiao, F. Jiang, Microbiome, 6, 211 (2018)

19. J. Lu, U. Idris, B. Harmon, J.J. Maurer, M.D. Lee, C. Hofacre, Appl Environ. Microbiol, 69 (2003)

20. M. Chichlowski, J. Croom, B.W. McBride, G.B. Havenstein, M.D. Koci, International Journal of Poultry Science, 6, 694-704 (2007)

21. D. Pan, Z. Yu, Gut Microbes, 5, 108-19 (2014)

22. C. Sun, S. Zhang, F.X. Xin, S. Shanmugam, Biotechnol Biofuels, 11, 42 (2018) 